\author{
(․ Е.Н. Дудинская, Н.В. Браилова, В.А. Кузнецова, О.Н. Ткачева
}

ФГАОУ ВО «Российский национальный исследовательский медицинский университет им. Н.И. Пирогова» Министерства здравоохранения Российской Федерации, Москва, Россия.

Остеопороз у лиц пожилого и старческого возраста можно сравнить с эпидемией 21 века в связи с большой распространенностью и повышением заболеваемости среди людей, переживших 50-летний порог, составляющих основную массу пациентов. Остеопороз связан со значительным повышением риска падений и переломов, приводящих к адинамии и увеличению риска смерти. Несмотря на недостаточную изученность патогенеза заболевания, имеющиеся данные уже позволили разработать меры профилактики и принципы лечения. В настоящее время существуют профилактические и лечебные мероприятия, направленные на снижение риска падений, переломов и повторных переломов, однако раннее выявление заболевания в пожилом возрасте достаточно часто затруднено в связи с характерными особенностями больных гериатрического профиля. Полиморбидность, стертость клинической картины, развитие старческой астении, саркопении, социально-психическая дезадаптация и повышение частоты депрессии делают популяцию людей пожилого и старческого возраста уязвимой в отношении повышения риска развития остеопороза, падений и переломов и ассоциированных с ними госпитализаций и смертности. В данном обзоре освещаются особенности патогенеза, клинической картины, принципов лечения и профилактики остеопороза в старшей возрастной группе.

КЛЮЧЕВЫЕ СЛОВА: старение; остеопороз; переломы; костная ткань; витамин D

\title{
OSTEOPOROSIS IN THE ELDERLY
}

(c) Ekaterina N. Dudinskaya, Natalia V. Brailova, Veronika A. Kuznetsova, Olga N. Tkacheva

${ }^{1}$ Pirogov Russian National Research Medical University, Moscow, Russia

Osteoporosis in the elderly and senile can be compared with the epidemic of the 21 st century due to the high prevalence and increased incidence among people who have survived the 50-year threshold, which make up the bulk of patients. Osteoporosis is associated with a significant increase in the risk of falls and fractures, leading to adynamia and an increased risk of death. Despite the insufficient knowledge of the pathogenesis of the disease, the available data have already allowed the development of preventive measures and treatment principles. Currently, there are preventive and therapeutic measures aimed at reducing the risk of falls, fractures and repeated fractures, however, earlier detection of the disease in old age is often difficult due to the characteristic features of geriatric patients. The polymorbidity, unexpressed clinical picture, the development of frailty syndrome, sarcopenia, social and mental maladaptation and an increase in the frequency of depression make the population of elderly and senile people vulnerable to an increased risk of osteoporosis, falls and fractures and associated hospitalizations and mortality. This review highlights the features of pathogenesis, clinical features, principles of treatment and prevention of osteoporosis in the older age group.

KEYWORDS: aging; osteoporosis; fractures; bone; vitamin D

\section{ВВЕДЕНИЕ}

За последние столетия значительно увеличилась ожидаемая продолжительность жизни во всем мире, что привело к появлению новых задач перед современной медициной. Необходимо глубокое изучение процессов старения, знание которых позволит улучшить качество жизни пожилых пациентов. Пациенты пожилого и старческого возраста, нуждающиеся в уходе, отнесены к группе старческой астении, которая включает в себя различные параметры, определяемые как самим пациентом при помощи специальных гериатрических шкал и опросников (потеря веса, слабость, утомляемость, снижение физической активности), так и при участии медицинского работника с помощью специальных тестов, оценивающих, в частности, скорость передвижения, снижение силы кисти [1]. Различные гериатрические синдромы, выявляемые у пожилых людей, взаимосвязаны между собой [2]. Так, потеря веса или саркопения характеризуются не только снижением мышечной массы, но и впоследствии развитием остеопороза. Остеопороз характеризуется низкой минеральной плотностью кости (МПК) и нарушением микроархитектуры костной ткани, что приводит к повышенной хрупкости костей и увеличению частоты низкотравматичных переломов у пациентов пожилого и старческого возраста. Кость является динамичной структурой, и на протяжении большей части жизни (за исключением детского и старческого возраста) 
в ней поддерживаются в равновесии процессы остеосинтеза и резорбции костной ткани. Какие-либо нарушения в процессе остеосинтеза приводят к снижению МПК. Несмотря на то что остеопороз по клиническим рекомендациям считается системным заболеванием, существует также и локальный остеопороз, который чаще всего является частым поттравматическим осложнением. Системный остеопороз подразделяется на первичный (наследственный, ювенильный, идиопатический, инволютивный) и вторичный. Большую часть всех системных остеопорозов составляет первичный инволютивный остеопороз (около 85\%), который делится на постменопаузальный (І тип) и на сенильный (II тип) [3, 4]. Диагноз сенильного остеопороза устанавливается преимущественно у лиц старше 70 лет.

\section{ЭТИОЛОГИЯ И ПАТОГЕНЕЗ СЕНИЛЬНОГО ОСТЕОПОРОЗА}

Старческий, или сенильный, остеопороз зависит от различных факторов: самого процесса старения, наличия сопутствующих заболеваний, образа жизни, физической активности, лекарственной терапии, гормонального статуса. Сенильный остеопороз поражает в равной степени как женщин, так и мужчин, что было подтверждено во многих исследованиях, например, в Роттердамском исследовании [5], проведенном в 2004 г. Сенильный остеопороз связан не только с усилением активности остеокластов, но и с уменьшением количества и активности остеобластов, что замедляет образование кости и приводит к уменьшению общей костной массы.

В настоящее время известно, что хроническое воспаление, являющееся важным механизмом инициации и развития старения, влияет и на процессы, протекающие в костной ткани. Так, значительную роль в ремоделировании кости играют провоспалительные цитокины, такие как ИЛ-6, ФНО-а, ИЛ-1, среди которых ИЛ-6 и ИЛ-1 являются мощными стимуляторами дифференцировки и активации остеокластов, что приводит к активной резорбции кости, а ФНО-а не только стимулирует резорбцию костной ткани, но и ингибирует образование новой кости. Кроме того, через воздействие ИЛ-1 и ФНО-а активируется индуцируемый путь синтеза оксида азота. Этот путь синтеза оксида азота ингибирует продукцию новых остеобластов и может индуцировать апоптоз остеобластов.

С возрастом происходят определенные гормональные изменения: меняется уровень гормона роста, половых гормонов и инсулиноподобного фактора роста (ИФР). Было отмечено у людей старого и пожилого возраста повышение уровня ИФР-связывающего белка, снижающего биодоступность ИФР и нейтрализующего его функциональную активность. В норме ИФР увеличивает количество активных остеобластов через стимуляцию пролиферации и дифференцировки мезенхимальных клеток костного мозга в остеобласты.

Значительный вклад в патогенез старения кости вносит дефицит витамина D, нарастающий с возрастом. В отсутствие нарушений метаболизма в организме 1,25-дигидроксивитамин D3, или кальцитриол (активная форма витамина D), обеспечивает всасывание кальция в кишечнике, снижение уровня паратгормона (путем прямого и опосредованного воздействия), приводя таким образом к снижению резорбции костной ткани. Соответственно, даже легкий дефицит витамина D может привести к вторичному гиперпаратиреозу с последующим развитием остеопороза. Снижение содержания в организме витамина D в пожилом возрасте может быть связано не только со снижением потребления пищи, содержащей витамин D, по причине снижения аппетита, социально-экономических факторов, изменения вкусовых особенностей пациента, но и замедлением его образования из провитамина D3 в коже, нарушением синтеза активной формы витамин D (1,25-дигидроксивитамин D3) в почках, которые также вовлекаются в процесс старения [6]. В связи с ухудшением всасывания кальция в кишечнике у людей старше 65 лет и в результате - снижением его уровня в сыворотке крови, по механизму отрицательной обратной связи увеличивается выработка паратгормона, который для нормализации уровня кальция в крови вымывает его из костей, активируя процесс резорбции костной ткани [7].

Характерной чертой сенильного остеопороза является равномерность поражения костной ткани: губчатой кости в осевом скелете, кортикальной кости в конечностях. В результате при сенильном остеопорозе наиболее характерные места переломов - позвонки (клиновидные безболевые переломы) и шейка бедренной кости, тогда как при постменопаузальном остеопорозе поражается губчатая кость, и наблюдаются компрессионные переломы позвонков, сопровождающиеся сильной болью [3, 7].

\section{ФАКТОРЫ РИСКА ОСТЕОПОРОЗА}

Диагностировать остеопороз до развития осложнений заболевания, то есть до того, как произойдет перелом, - достаточно трудная задача, однако, зная факторы риска, остеопороза и переломов, можно наблюдать пациентов, входящих в группу риска и предотвратить в дальнейшем прогрессирование заболевания. Ранее выделяли факторы риска развития остеопороза: модифицированные и немодифицированные, однако в настоящее время в группе всех женщин в постменопаузальном периоде и мужчин старше 50 лет рекомендуется оценивать индивидуальную 10-летнюю вероятность низкотравматичного перелома с использованием алгоритма FRAX (fracture risk assessment tool), который учитывает вероятность летального исхода от других причин (табл. 1) [4, 8]. Несмотря на то что данный алгоритм рекомендован ВО3, он имеет некоторые недостатки, так как не учитываются такие условия, актуальные для людей старческого и пожилого возраста, как, например, противораковое лечение, возраст старше 90 лет, количество выкуриваемых сигарет, локализация перенесенного перелома, наличие старческой астении. Также в FRAX не учитывается поражение почек и степень снижения их функции, но, несмотря на это, стоит обращать особое внимание на пациентов, находящихся на диализе, так как данная процедура значительно повышает риск переломов. Кроме того, стоит отметить, что при помощи алгоритма FRAX не предоставляется возможным оценить пациентов, ранее получавших и получающих терапию по поводу остеопороза (за исключением пациентов, не принимающих терапию более 2 лет) [3, 4, 8, 9]. 
Таблица 1. Факторы риска остеопороза

\begin{tabular}{|c|c|c|c|}
\hline \multicolumn{2}{|c|}{$\begin{array}{c}\text { Основные факторы риска остеопороза } \\
\text { и переломов костей }\end{array}$} & \multicolumn{2}{|c|}{$\begin{array}{c}\text { Клинические факторы риска, включенные } \\
\text { в инструмент FRAX }\end{array}$} \\
\hline $\begin{array}{c}\text { Модифицируемые факторы } \\
\text { риска }\end{array}$ & $\begin{array}{l}\text { Немодифицируемые } \\
\text { факторы риска }\end{array}$ & Возраст & Ревматоидный артрит \\
\hline $\begin{array}{c}\text { Системный прием ГК } \\
\text { более трех месяцев (A) }\end{array}$ & $\begin{array}{c}\text { Возраст } \\
\text { старше } 65 \text { лет (A) }\end{array}$ & Пол & $\begin{array}{c}\text { Вторичные причины } \\
\text { остеопороза: СД } 1 \text { типа, } \\
\text { несовершенный остеогенез } \\
\text { взрослых, длительно } \\
\text { нелеченый тиреотоксикоз, } \\
\text { гипогонадизм или } \\
\text { преждевременная } \\
\text { менопауза (<40 лет), } \\
\text { хроническое недоедание } \\
\text { или мальабсорбция } \\
\text { и хроническая болезнь } \\
\text { печени }\end{array}$ \\
\hline Табакокурение (A) & Женский пол (А) & $\begin{array}{c}\text { Предшествующий } \\
\text { остеопоротический } \\
\text { перелом (включая } \\
\text { переломы позвонков } \\
\text { с клинической картиной } \\
\text { и без нее) }\end{array}$ & $\begin{array}{c}\text { Переломы бедра } \\
\text { у родителей в анамнезе }\end{array}$ \\
\hline $\begin{array}{c}\text { Недостаточное } \\
\text { потребление кальция (A) }\end{array}$ & $\begin{array}{c}\text { Белая (европеоидная) раса } \\
\text { (В) }\end{array}$ & МПК шейки бедра & $\begin{array}{c}\text { Курение } \\
\text { (на данный момент) }\end{array}$ \\
\hline Дефицит витамина D (A) & $\begin{array}{l}\text { Предшествующие } \\
\text { переломы (A) }\end{array}$ & 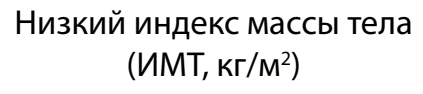 & $\begin{array}{c}\text { Прием алкоголя } \\
\text { (3 или более порций/сут) }\end{array}$ \\
\hline $\begin{array}{l}\text { Злоупотребление } \\
\text { алкоголем (A) }\end{array}$ & Низкая МПК (А) & \multicolumn{2}{|c|}{$\begin{array}{c}\text { Пероральный прием глюкокортикоидов } \geq 5 \text { мг/сут } \\
\text { в эквиваленте преднизолона в течение >3 месяцев } \\
\text { (вне зависимости от давности) }\end{array}$} \\
\hline $\begin{array}{c}\text { Низкая физическая } \\
\text { активность (В) }\end{array}$ & Склонность к падениям (А) & & \\
\hline \multirow[t]{4}{*}{$\begin{array}{c}\text { Длительная } \\
\text { иммобилизация (B) }\end{array}$} & $\begin{array}{l}\text { Наследственность } \\
\text { (семейный анамнез } \\
\text { остеопороза) (А) }\end{array}$ & & \\
\hline & $\begin{array}{c}\text { Гипогонадизм у мужчин } \\
\text { и женщин* (A) }\end{array}$ & & \\
\hline & $\begin{array}{c}\text { Снижение клиренса } \\
\text { креатинина и/или } \\
\text { клубочковой фильтрации } \\
\text { (В) }\end{array}$ & & \\
\hline & $\begin{array}{c}\text { ИМТ <20 кг/м² и/или } \\
\text { вес менее } 57 \text { кг (А) }\end{array}$ & & \\
\hline
\end{tabular}

Примечания: МПК - минеральная плотность кости; ИМТ - индекс массы тела; * - низкий уровень тестостерона у мужчин с соответствующей симптоматикой и ранняя менопауза у женщин (ранее 45 лет).

\section{ОСОБЕННОСТИ ГЕРИАТРИЧЕСКИХ БОЛЬНЫХ}

С возрастом происходят структурная перестройка и инволютивные процессы в различных органах и тканях, снижается репаративная функция организма, что значительно уменьшает функциональные способности органов и тканей и создает определенные трудности при работе с пациентами пожилого и старческого возраста. Характерной чертой пожилых пациентов и одной из самых значимых проблем, ухудшающих диагностику, лечение и профилактику остеопороза, является поли- морбидность. Наличие многочисленной разнообразной симптоматики сопутствующих заболеваний зачастую не позволяет быстро распознать остеопороз и его осложнения. Переломы часто являются случайной находкой на рентгенограмме, например, клиновидный перелом тела позвонка, который может долгое время скрываться под маской остеохондроза. Возможны обратные случаи, когда при недиагностированной онкопатологии метастазы в области тела позвонка с переломом изначально могут быть расценены как проявление остеопороза. Таким образом, при полиморбидности возможна как 
гиподиагностика, так и гипердиагностика заболевания, может наблюдаться сочетание остеопороза с другими заболеваниями со сходной клинической картиной и взаимным отягощением течения друг друга.

Для гериатрических больных характерны стертость течения многих заболеваний, присутствие неклассических симптомов или их полное отсутствие. Остеопороз у пожилого пациента без каких-либо жалоб при переломе позвонка можно сравнить с течением пневмонии без сильного повышения температуры и кашля. Стертость клинической картины затрудняет диагностический поиск, а пациент обращается за помощью к специалисту на более поздних стадиях при развитии осложнений.

Синдром старческой астении, выявляемый в старшей возрастной группе, тесно ассоциирован с развитием саркопении, остеопороза и повышением частоты падений и переломов в 1,2-2,8 раза. Пациенты со старческой астенией в 1,2-1,8 раза чаще подвергаются госпитализациям в сравнении с общей популяцией людей пожилого и старческого возраста [2]. Характерной чертой гериатрических пациентов, госпитализированных в стационар, является возрастание риска падений при увеличении койко-дней в стационаре, повышение вероятности заражения внутрибольничными инфекциями, тяжело поддающимися лечению.

Следующая особенность пациентов гериатрического профиля - социально-психическая дезадаптация, связанная со значительными изменениями в жизни пациента: выход на пенсию, снижение социальной и физической активности, часто ухудшение экономического положения. В результате перечисленных причин у пожилого пациента может развиться депрессия [10].

\section{ПАДЕНИЯ И ПЕРЕЛОМЫ У ПОЖИЛЫХ}

У молодых лиц падение обычно не приводит к каким-либо серьезным последствиям в отличие от пожилых пациентов. У пациентов пожилого и старческого возраста падения могут быть вызваны различными причинами, обусловленными неврологическими нарушениями, плохо контролируемым уровнем глюкозы крови при сахарном диабете, использованием некоторых лекарственных препаратов, например, бензодиазепинов, головокружениями различного генеза, различными заболеваниями нижних конечностей. Падения в пожилом возрасте чаще, чем у более молодых лиц, приводят к переломам, снижающим физическую активность пациентов и увеличивающим риск смертности. Переломы у пациентов гериатрического профиля могут происходить даже при нормальной МПК, поэтому чем старше пациент, тем падения становятся все более значимым фактором риска переломов. Основные факторы риска переломов можно разделить на внутренние (пол, возраст и т.д.) и внешние (слабое освещение в помещении, кресла без ручек, высокие ступени и др.) [4]. При сравнении пациента, у которого снижена МПК, но отсутствуют другие факторы риска или они минимальны, и пациента с более чем четырьмя факторами риска и нормальной МПК, считается, что у второго риск падения выше, однако риск перелома остается выше у первого. Соответственно, важной задачей лечащего врача становится профилактика падения и повышение МПК. Однако если перелом случился, то данному пациенту, согласно клиническим рекомендациям, выставляется диагноз остеопороза и начинается лечение, независимо от результатов денситомерии или FRAX (если отсутствуют другие заболевания опорной системы) [8].

Существенная разница между заживлением перелома у молодых и у пожилых пациентов связана с возрастным ухудшением процессов восстановления поврежденной костной ткани. У пожилых людей нарушается и снижается не только остеогенная дифференцировка, но и ангиогенез вследствие уменьшения активности эндотелиальных клеток гемостатического пути, факторов роста, нейрохимических медиаторов. При нарушении или ограничении ангиогенеза и нестабильности перелома происходит активация другого механизма - эндохондральное окостенение с проникновением сосудов и клеток-предшественниц из мезенхимы в новообразованную хондрогенную ткань, что приводит к ухудшению заживления перелома и формирования кортикальной кости.

Минерализация и прочность костной ткани оцениваются по нескольким параметрам при проведении денситометрии: костный минеральный компонент (КМК) и МПК. Оба эти параметра характеризуют количество минерализованной костной ткани, однако КМК определяется длиной сканирующего пути (г/см), а МПК определяет количество костной ткани в сканируемой

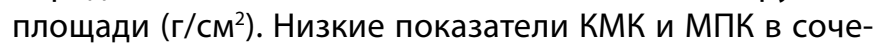
тании с первым переломом могут запускать «каскад переломов» $[4-9,11]$. Женщины с большей вероятностью, чем мужчины, переносят первый и все последующие переломы. Согласно Рейкьявикскому исследованию, структура повторных переломов была похожа на первый, при этом риск второго перелома сразу после первого был существенно высок, снижался со временем, однако с каждым годом отмечалось умеренное увеличение риска повторного перелома на 5\%. Через год после первого перелома риск повторного был в 2,7 раза выше, чем в общей популяции, при этом статистически значимой разницы между мужчинами и женщинами выявлено не было. Наряду с повторными переломами большой проблемой является хроническая боль, которая присутствует у 75\% пациентов, в связи с которой многие ежедневно принимают нестероидные противовоспалительные препараты [7, 12].

\section{ПРИНЦИПЫ ЛЕЧЕНИЯ ОСТЕОПОРОЗА У ПОЖИЛЫХ}

Перед назначением лечения пациентам пожилого и старческого возраста необходимо тщательно оценить пользу и риск назначаемой терапии. Подобная осторожность связана с более высокой частотой лекарственных осложнений у пожилых людей по сравнению с больными молодого и среднего возраста, с полипрагмазией в данной возрастной группе, в связи с чем назначение длительного медикаментозного лечения негативно сказывается на приверженности пациента к терапии, с возрастным снижением чувствительности рецепторов, что может повлиять на фармакодинамику препарата. С пациентом и по возможности с родственниками должна быть проведена беседа о необходимости назначаемой терапии, ее рисках и осложнениях [10]. 
Лечение остеопороза имеет несколько основных направлений - увеличение прочности костной ткани, снижение или полное прекращение потери костной массы, нормализация процессов костного ремоделирования, снижение риска падения и переломов, улучшение функциональной активности пациента. В первую очередь важным компонентом лечения пациентов с остеопорозом является немедикаментозное лечение, основанное на изменении образа жизни. Некоторым пациентам для повышения МПК при небольшом его снижении достаточно скорректировать питание и увеличить время пешей прогулки на полчаса. Существуют обучающие программы и школы остеопороза, информирующие пациентов об остеопорозе и мерах профилактики первичных и повторных переломов $[4,10]$.

Препараты кальция и витамина D, содержащие колекальциферол, являются обязательным компонентом терапии остеопороза наряду с патогенетической терапией, оказывая положительное влияние на баланс и мышечную силу и снижая, таким образом, риск падений и переломов [3]. Активные метаболиты витамина D или их аналоги могут быть назначены пациентам с выраженной гипокальциемией или терминальной хронической почечной недостаточностью по абсолютным показаниям или пожилым пациентам со скоростью клубочковой фильтрации меньше 60 мл/мин и высоким риском падений по относительным показаниям. Прием данных препаратов существенно увеличивает всасывание кальция в кишечнике, и снижает уровень паратгормона в крови и требует частого мониторирования уровня кальция в сыворотке крови и моче. Сенильному остеопорозу может сопутствовать синдром мальабсорбции, когда ухудшается всасывание кальция и снижается чувствительность к рецепторам витамина $D$, что определяет необходимость назначения активных метаболитов витамина D также в данной группе пациентов $[3,4,6]$.

В качестве базовой медикаментозной патогенетической терапии используют препараты, которые условно можно поделить на антирезорбтивные, которые подавляют костную резорбцию через воздействие на остеокласты, увеличивают минерализацию костной ткани, и анаболические, которые увеличивают образование костной ткани, повышая костную массу и улучшая архитектонику кости. К антирезорбтивным препаратам относятся бисфосфонаты, селективные модуляторы эстрогеновых рецепторов, деносумаб. Анаболическим препаратом, представленным в отечественной практике, является терипаратид. Для лечения остеопороза у мужчин применяются азотсодержащие бисфосфонаты (алендронат, золедроновая кислота), деносумаб и терипаратид. Для лечения постменопаузального остеопороза можно также использовать ряд препаратов из группы бисфосфонатов - ризедронат, ибандронат.

Выбор в пользу того или иного препарата зависит от тяжести остеопороза, наличия противопоказаний, предпочтений пациента. Так, в случае бисфосфонатов возможно внутривенное и пероральное применение препаратов. Однако нет данных об эффективности ибандроната в профилактике переломов у пожилых людей [3]. Применение золедроновой кислоты в исследовании HORIZON Pivotal Fracture Talk (HORIZON-PFT) доказало эффективность у женщин в постменопаузе со средним возрастом 73 года в снижении риска переломов позвонков, бедер и внепозвоночных переломов. В опубликованном в 2010 г. сводном анализе исследований HORIZON PFT и HORIZON-RFT снижение риска перелома бедра не соответствовало статистической значимости у пациентов $\geq 75$ лет, что могло быть связано с недостаточным объемом выборки или влиянием других внескелетных факторов риска на переломы бедра, увеличивающихся с возрастом. Ризедронат также не подтвердил значимое снижение риска переломов бедра в группе лиц старше 80 лет. Полученные данные могут быть связаны с тем, что бисфосфонаты, повышая МПК, не влияют на внескелетные факторы риска переломов, такие как нарушения походки, равновесия и риск падения, значимые в пожилой группе [3].

Деносумаб продемонстрировал по результатам исследования FREEDOM у лиц в возрасте $\geq 75$ лет безопасность и значительное снижение риска переломов бедра на $62 \%$, позвонков и внепозвоночных переломов до той же степени, что и у более молодых лиц [3].

Применение стронция ранелата у женщин со средним возрастом 76,7 года снизило через 5 лет риск переломов позвонков и внепозвоночных переломов на $31 \%$, однако наличие повышенного риска сердечнососудистых событий, в т.ч. инфаркта миокарда, и венозной тромбоэмболии, не позволяет его применять у лиц старше 75 лет [3].

Терипаратид показан для лечения преимущественно тяжелых форм остеопороза (один или более переломов тел позвонков, перелом проксимального отдела бедра, множественные повторные переломы костей скелета) с продолжительностью лечения до 18-24 месяцев, после чего может быть продолжена терапия бисфосфонатами (алендронат), так как данная схема приводит к дальнейшему увеличению МПК у женщин в постменопаузальном периоде [4, 7, 13]. Однако анализ, проведенный в группе лиц старше 75 лет по изучению влияния терипаратида выявил, что риск переломов позвонков и внепозвоночных переломов статистически значимо не различался у молодых и пожилых пациентов [14]. Не было существенной разницы и в переносимости препарата. Таким образом, возраст не влияет на безопасность и эффективность терипаратида в профилактике переломов. Необходимость ежедневного подкожного введения препарата создает определенные трудности для пожилых людей. Другой анаболический препарат, абалопаратид, продемонстрировал в сравнительном исследовании ACTIVE последовательное снижение риска переломов позвонков и внепозвоночных переломов в разных возрастных группах, но он пока не зарегистрирован в России [15].

\section{МЕРЫ ПРОФИЛАКТИКИ ОСТЕОПОРОЗА}

Самым важным в практике врача-гериатра является предотвращение развития заболевания. Для этого в первую очередь важен образ жизни пациента. Если у больного не присутствует необходимый минимум физической нагрузки и нет соответственного его состоянию рациона питания, то резко возрастают риски развития остеопороза, падений и переломов. При отсутствии необходимой физической нагрузки у пациента снижается мышечная сила, а редкая избыточная активность лишь 
оказывает избыточное давление на кости скелета. Для поддержания нормальной плотности костной ткани нужна умеренная регулярная физическая нагрузка. Так, например, из физических нагрузок самым эффективным видом является ходьба и некоторые упражнения на равновесие и увеличение мышечной силы, однако запрещаются прыжки и бег, которые могут спровоцировать падение. В рацион необходимо включить продукты, богатые кальцием, магнием, и полностью отказаться от курения и употребления алкоголя. Для тех, у кого имеется высокий риск развития перелома бедра, рекомендуется ношение постоянного бедренного протектора.

Служба профилактики повторных переломов в первую очередь направлена на предотвращение падений. Для снижения риска падений в первую очередь стоит лечить или контролировать сопутствующие заболевания (гипертоническая болезнь, анемия, сахарный диабет, неврологические заболевания и др.). С пациентом следует провести беседу о том, что различные внешние факторы (высокие ступеньки и др.) могут привести к падению и в дальнейшем сильно снизить качество жизни. Для уменьшения риска падения пациент может пользоваться тростью, ему следует носить более устойчивую обувь на низкой платформе или низком каблуке.

С низкой МПК почти всегда связана саркопения, которая соответствует более низкой способности выполнять повседневную нагрузку. В совокупности это может влиять на более высокую вероятность развития осложнений после перелома, увеличения затраты ресурсов здравоохранения. И в данном случае самой лучшей профилактикой являются оптимизация статуса питания и восполнение дефицита питательных веществ, в частности увеличение содержания белка в питании до 1,5 г/кг массы тела. После произошедшего перелома для врача важной задачей является эффективное обезболивание пациента. Не только нестероидные противовоспалительные препараты, но и терипаратид влияют на снижение болевого синдрома в области спины. Необходимо быстрое назначение медикаментозной антиостеопоротической терапии, максимально краткосрочная иммобилизация, после которой должны проводиться физиотерапевтическое лечение и ранняя активизация пациента уже на этапе постельного режима. Все проводимые процедуры должны быть согласованы с врачом-травматологом $[3,4,8,16]$.

\section{ЗАКЛЮЧЕНИЕ}

В пожилом возрасте распространенность остеопороза и переломов, ассоциированных с ним, увеличивается, особенно среди пациентов со старческой астений. Значимое социально-экономическое, медицинское бремя переломов у пожилых людей требует обязательной повсеместной диагностики остеопороза, переломов и незамедлительного лечения в данной возрастной группе. Наряду с применением кальция и витамина D, являющихся важными компонентами в лечении остеопороза в пожилом возрасте, необходимо обязательное патогенетическое лечение, которое еще больше будет снижать риск переломов, особенно переломов позвонков. У пожилых людей со старческой астенией с установленным остеопорозом лечение может быть даже более эффективным, чем у более молодых пациентов.

\section{ДОПОЛНИТЕЛЬНАЯ ИНФОРМАЦИЯ}

Конфликт интересов. Авторы декларируют отсутствие явных и потенциальных конфликтов интересов, связанных с публикацией настоящей статьи.

\section{СПИСОК ЛИТЕРАТУРЫ | REFERENCES}

1. Dent E, Morley JE, Cruz-Jentoft AJ, et al. Physical Frailty: ICFSR International Clinical Practice Guidelines for Identification and Management. J. Nutr. Health Aging. 2019;23(9):771-787. doi: https/doi.org/10.1007/s12603-019-1273-z.

2. Ткачева О.Н., Рунихина Н.К., Котовская Ю.В., и др. Ведение пациентов со старческой астенией в первичном звене здравоохранения. Учебное пособие для врачей - М.: Изд-во РАМН, 2016. [Tkacheva ON, Runikhina NK, Kotovskaya YuV, et al. Vedenie pacientov so starcheskoi asteniei v pervichnom zvene zdravooxranenia. Uchebnoe posobie dlya vrachei. Moscow: Izd-vo RAMN; 2016. (In Russ).].

3. Gielen $\mathrm{E}$, Bergmann $\mathrm{P}$, Bruyère $\mathrm{O}$, et al. Osteoporosis in Frail Patients: A Consensus Paper of the Belgian Bone Club. Calcif. Tissue Int. 2017;101(2):111-131. doi: https/doi.org/10.1007/s00223-017-0266-3.

4. Клинические рекомендации по профилактике и ведению больных с остеопорозом. Издание 2-ое, дополненное. / Под ред. Лесняк О.М. - Ярославль: ИПК «Литера», 2013. [Lesnyak OM, editor. Klinicheskie rekomendacii po profilaktike i vedeniju bol'nyh s osteoporozom. Izdanie 2-oe, dopolnennoe. Yaroslavl': IPK «Litera»; 2013 (In Russ).].

5. de Liefde II, van der Klift M, de Laet CEDH, et al. Bone mineral density and fracture risk in type-2 diabetes mellitus: the Rotterdam Study. Osteoporos. Int. 2005;16(12):1713-1720. doi: https/doi.org/10.1007/s00198-005-1909-1.

6. Пигарова Е.А., Рожинская Л.Я., Белая Ж.Е., и др. Клинические рекомендации Российской ассоциации эндокринологов по диагностике, лечению и профилактике дефицита витамина D у взрослых // Проблемы Эндокринологии. - 2016. - Т. 62. №4. - C. 60-84. [Pigarova EA, Rozhinskaya LY, Belaya JE, et al.
Russian Association of Endocrinologists recommendations for diagnosis, treatment and prevention of vitamin D deficiency in adults. Problems of Endocrinology. 2016;62(4):60-84. (In Russ).] doi: https/doi.org/10.14341/probl201662460-84.

7. Lau AN, Adachi JD. Bone Aging. In: Nakasato Y, Yung R, editors. Geriatric Rheumatology. New York: Springer, NY; 2011, p.11-17. Doi: https://doi.org/10.1007/978-1-4419-5792-4.

8. Мельниченко Г.А., Белая Ж.Е., Рожинская Л.Я., и др. Краткое изложение клинических рекомендаций по диагностике и лечению остеопороза // Остеопороз и остеопатии. - 2016. T. 19. — №3. - C. 28-36. [Melnichenko GA, Belaya ZE, Rozhinskaya LY, et al. Summary of Clinical Guidelines for the Diagnosis and Treatment of Osteoporosis of the Russian Association of Endocrinologists. Osteoporosis and Bone Diseases. 2016;19(3):28-36. (In Russ).]. doi: https/doi.org/10.14341/osteo2016328-36.

9. Karlsson MK, Magnusson H, von Schewelov T, Rosengren BE. Prevention of falls in the elderly - a review. Osteoporos. Int. 2013;24(3):747-762. doi: https/doi.org/10.1007/s00198-012-2256-7.

10. Рожинская Л.Я. Диагностика и лечение остеопороза // Клиническая геронтология. - 2007. - Т. 13. - №2. - С. 37-46. [Rozhinskaja LYa. Diagnostika i lechenie osteoporoza // Klinicheskaja gerontologija. 2007;13(2):37-46 (In Russ).].

11. Qi H, Sheng Y, Chen S, et al. Bone mineral density and trabecular bone score in Chinese subjects with sarcopenia. Aging Clin. Exp. Res. 2019;31(11):1549-1556 doi: https/doi.org/10.1007/s40520-019-01266-8.

12. Johansson H, Siggeirsdóttir K, Harvey NC, et al. Imminent risk of fracture after fracture. Osteoporos. Int. 2016;28(3):775-780. doi: https/doi.org/10.1007/s00198-016-3868-0. 
13. Мазуров В.И., Лесняк О.М., Белова К.Ю., и др. Алгоритмы выбора терапии остеопороза при оказании первичной медико-санитарной помощи и организации льготного лекарственного обеспечения отдельных категорий граждан, имеющих право на получение государственной социальной помощи. Системный обзор и резолюция Экспертного совета Российской ассоциации по остеопорозу // Профилактическая медицина. — 2019. — №1. - C. 57-65. [Mazurov VI, Lesnyak OM, Belova KY, et al. Algorithm for selection of drug for osteoporosis treatment in primary care and in organization of provision with medicinal products of citizens eligible for state social assistance. Review of the literature and position of Russian Association on Osteoporosis Expert Council. Profilakticheskaya meditsina. 2019;22(1):57. (In Russ.)] doi: https/doi.org/10.17116/profmed20192201157.
14. Boonen S, Marin F, Mellstrom D, et al. Safety and Efficacy of Teriparatide in Elderly Women with Established Osteoporosis: Bone Anabolic Therapy from a Geriatric Perspective. J. Am. Geriatr. Soc. 2006;54(5):782-789. doi: https/doi.org/10.1111/j.1532-5415.2006.00695.x.

15. Cosman F, Hattersley G, Hu M-y, et al. Effects of AbaloparatideSC on Fractures and Bone Mineral Density in Subgroups of Postmenopausal Women With Osteoporosis and Varying Baseline Risk Factors. J. Bone Miner. Res. 2017;32(1):17-23. doi: https/doi.org/10.1002/jbmr.2991.

16. Fahrleitner-Pammer A, Langdahl BL, Marin F, et al. Fracture rate and back pain during and after discontinuation of teriparatide: 36-month data from the European Forsteo Observational Study (EFOS). Osteoporos. Int. 2010;22(10):2709-2719. doi: https/doi.org/10.1007/s00198-010-1498-5.

\section{ИНФОРМАЦИЯ ОБ АВТОРАХ [AUTHORS INFO]}

*Браилова Наталия Васильевна, К.M.H. [Natalia V. Brailova, MD, PhD]; адрес: Россия, 129226, г. Mосква, ул. 1-я Леонова, д. 16 [address: 16 1-st Leonova street, 129226 Moscow, Russia]; ORCID: 0000-0002-1216-0787; eLibrary SPIN: 2900-8384; e-mail: n.kokshagina@mail.ru.

Дудинская Екатерина Наильевна, к.м.н. [Ekaterina N. Dudinskaya, MD, PhD]; e-mail: katharina.gin@gmail.com; ORCID: 0000-0001-7891-6850; eLibrary SPIN: 4985-6315.

Кузнецова Вероника Андреевна, студентка [Veronika A. Kuznetsova, Student]; e-mail: nik_k_ot@mail.ru; ORCID: 0000-0003-0816-4870; eLibrary SPIN: 86174030.

Ткачева Ольга Николаевна, д.м.н., професcop [Olga N. Tkacheva, MD, PhD, professor]; e-mail: tkacheva@rgnkc.ru; ORCID: 0000-0002-4193-688X; eLibrary SPIN: 677476;

\section{ЦИТИРОВАТЬ:}

Дудинская Е.Н., Браилова Н.В., Кузнецова В.А., Ткачева О.Н. Остеопороз у пожилых пациентов // Остеопороз и остеопатии. — 2019. - Т. 22. — №3 - C.34-40. doi: https://doi.org/10.14341/osteo12352

\section{TO CITE THIS ARTICLE:}

Dudinskaya EN, Brailova NV, Kuznetsova VA, Tkacheva ON. Osteoporosis in the elderly. Osteoporosis and bone diseases. 2019;22(3):34-40. doi: https://doi.org/10.14341/osteo12352 